\title{
Análise biomorfométrica da ostra-do-mangue cultivada no litoral amazônico
}

Biomorphometric relationships of the mangrove oyster cultivated on the amazonian coast

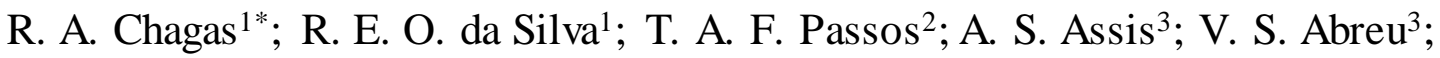 \\ W. C. R. Santos ${ }^{4}$; M. R. F. Barros ${ }^{2}$; M. Herrmann \\ ${ }^{1}$ Pós-Graduação em Ecologia (PPGEco/UFPA), Universidade Federal do Pará(UFPA), 66075-110, Belém - PA, \\ Brasil. \\ ${ }^{2}$ Pós-Graduação em Aquicultura e Recursos Aquáticos Tropicais (PPGAqRAT/UFRA), Universidade Federal Rural \\ da Amazônia (UFRA), 66077-530, Belém - PA, Brasil. \\ ${ }^{3}$ Universidade Federal Rural da Amazônia (UFRA), 66077-530, Belém - PA, Brasil. \\ ${ }^{4}$ Laboratório de Pesca e Biodiversidade Aquática (LPBAq/Cepnor) Universidade Federal Rural da Amazônia \\ (UFRA), 66077-530, Belém - PA, Brasil. \\ ${ }^{5}$ Instituto Socioambiental e dos Recursos Hídricos (ISARH), Universidade Federal Rural da Amazônia (UFRA), \\ 66077-530, Belém - PA, Brasil.
}

*rafaelanaisce@hotmail.com

(Recebido em 26 demaio de 2019; aceito em 31 de outubro de 2019)

\begin{abstract}
A ostreicultura surge no contexto mundial como uma das alternativas mais viáveis ao declínio da pesca e o fornecimento de recurso alimentício fresco. No Brasil, cultiva-se ostras do gênero Crassostrea e, no estado do Pará, cultiva-se a Crassostrea tulipa, conhecida por ostra-do-mangue. O presente estudo tem como objetivo caracterizar a biomorfometria da concha, estimar o Índice de Estabilização da Forma (IEF) da concha e o rendimento da carne comestível de C. tulipa, a partir de 1.028 ostras coletadas na ostreicultura da Associação de Agricultores, Pecuaristas e Aquicultores (ASAPAQ), situada no rio Urindeua, litoral amazônico, estado do Pará, no mês de abril de 2016. Realizou-se relações biomorfométricas entre a morfometria da concha (comprimento, largura e altura) e a biomassa (total e visceral), estimou-se o rendimento percentual da carne comestível e descreveu-se o IEF através de razões entre a morfometria da concha. Crassostrea tulipa apresenta excelentes relações biomorfométricas, gerando equações que satisfazem estimação de medidas morfométricas. Além disso, apresenta mais de $20 \%$ de rendimento da carne. A análise de IEF, indica uma tendência a estabilização da forma da concha ao atingir $60 \mathrm{~mm}$. Recomenda-se uma análise de IEF em ostras oriundas de ambientes naturais e sob influência da densidade de ostras e/o tipo de estrutura no qual a ostra está sendo cultivada. Este trabalho possibilita a estimação de carne de ostras comercializadas com base na mensuração da medida da altura da concha (mm) através da equação $B v=-4,29+1,94 A$.
\end{abstract}

Palavras-chave: Mollusca, Bivalvia, Ostreidae, Ostreicultura, IEF.

Oyster farming appears in the world context as one of the most viable alternatives to the decline of fishing and the provision of fresh food resources. In Brazil, the genus Crassostrea is used in oyster farming, and in the state of Pará, the oyster Crassostrea tulipa, known as mantras oyster, is used. The present study aims to characterize the shell biomorphometry, to estimate the Shell Shape Stabilization Index (IEF) and the yield of the C. tulipa edible meat, from 1,028 oysters collected in the oysterfarming of the Association of Farmers, Pecuaristas and Aquicultores (ASAPAQ), located in the Urindeua river, Amazonian coast, state of Pará, in April 2016. Biomorphometric relationships between the morphometry of the shell (length, width, and height) and biomass (total and visceral) were estimated, the percentage yield of edible meat was evaluated and the IEF was described through reasons between shell morphometry. C. tulipa presents excellent biomorphometric relationships, generating equations that satisfy the estimation of morphometric measurements. In addition, it has more than $20 \%$ of meat yield. The analysis of IEF indic ates a tendency to stabilize the shape of the shell when reaching $60 \mathrm{~mm}$. It is recommended an analysis of IEF in oysters from natural environments and under the influence of oyster density and/or the type of structure in which the oyster is being grown. Through this study it is possible to estimate oyster meat commercialized only by measuring the height of the shell $(\mathrm{mm})$ through the equation $B v=-4.29+1.94 A$.

Key words: Mollusca, Bivalvia, Ostreidae, Oyster farming, IEF. 


\section{INTRODUÇÃO}

O extrativismo descontrolado tem levado a uma redução mundial nos estoques de ostras [1,2]. Neste contexto, o cultivo de moluscos, torna-se a alternativa mais viável a fim de mitigar o declínio dos estoques naturais [3]. Em 2015, a malacocultura contribuiu com 15,5\% (16,5 milhões de toneladas) da produção total da aquicultura (continental, costeira e marinha) a nível mundial, movimentando US\$ 18 bilhões. Neste cenário, a ostreicultura corresponde a 32,4\% (cinco mil ton.) da produção, movimentando quatro bilhões de USD [4]. No Brasil, o cultivo de moluscos bivalves contribuiu, em 2016, com 1,5\% (21 mil ton.) da aquicultura nacional, movimentando R \$ 68,5 milhões. Neste mesmo ano, a ostreicultura do estado do Pará contribuiu com apenas 0,2\% (42 ton.) da produção nacional, gerando cerca de $\mathrm{R} \$ 322$ mil [5].

Dentre as ostras mais difundidas e comercializadas no mundo estão a ostra-plana-chilena, Ostrea edulis (Linnaeus, 1758), ostra norte-americana Crassostrea virginica (Gmelin, 1791) e a ostra introduzida Crassostrea gigas (Thunberg, 1793), conhecida como e ostra-japonesa [6]. Hernandez et al. (1998) [7] acrescentam a ostra-do-mangue Crassostrea rhizophorae (Guilding, 1828) como uma das espécies com maior perspectiva para o desenvolvimento da ostreicultura nos países tropicais da costa atlântica americana.

Atualmente, mesmo com os diversos estudos que discutem a taxonomia das ostras brasileiras, há uma divergência taxonômica das ostras nativas cultivadas $[8,9,10,11,12]$. Por conta disso, a nível mundial, o Brasil consta apenas como produtor de Crassostrea sp., ou seja, sem espécie especificada. A ostreicultura nacional está presente em todo litoral brasileiro. Entretanto, no Norte e Nordeste do país o cultivo acontece de forma artesanal enquanto que nas regiões Sul e Sudeste, de forma industrial [13, 14], com destaque ao estado de Santa Catarina, responsável por praticamente toda produção brasileira (97,9\%) [5]. No estado do Pará, a ostreicultura é recente e apresenta-se como uma atividade secundária para os pescadores artesanais e os agricultores familiares [13, 14, 15], principalmente pela dependência da produção e da coleta de sementes no meio ambiente, que não é contínua ao longo do ano [16].

Funo et al. (2015) [17] citam que é necessário intensificar os estudos sobre as espécies nativas de importância comercial, bem como forma de desenvolver técnicas de cultivo adequadas a cada região e otimizar os esforços dos produtores em cada etapa do processo de cultivo. Neste sentido, a ostreicultura apresenta estudos desde a década de 1970 [18], que possibilitaram seu rápido desenvolvimento. Dentre esses estudos, destacam-se a dinâmica reprodutiva (e.g., indução a desova, aspectos reprodutivos), do crescimento, além das influências ambientais no desenvolvimento da ostreicultura e eventuais predadores naturais [16, 19, 20, 21]. Entretanto, observa-se através da revisão bibliográfica efetuada, que não existe dados que possibilite a estimação da produção da biomassa (em toneladas) de ostra comercializa, pois, as estatísticas se delimitam a produção em dúzias.

Partindo do exposto, o presente trabalho tem como objetivo caracterizar as relações biomorfométricas e o rendimento da carne comestível da ostra-do-mangue Crassostrea tulipa (Lamarck, 1819) cultivada no litoral amazônico, Norte do Brasil.

\section{MATERIAL E MÉTODOS}

\section{1 Área de estudo}

O estudo desenvolveu-se na ostreicultura da Associação dos Agricultores, Pecuaristas e Aquicultores (ASAPAQ), localizado na zona estuarina da bacia hidrográfica do rio Urindeua, na vila de Santo Antônio de Urindeua (Figuras 1 e 2B), município de Salinópolis, estado do Pará, Amazônia Oriental, região Norte do Brasil. 

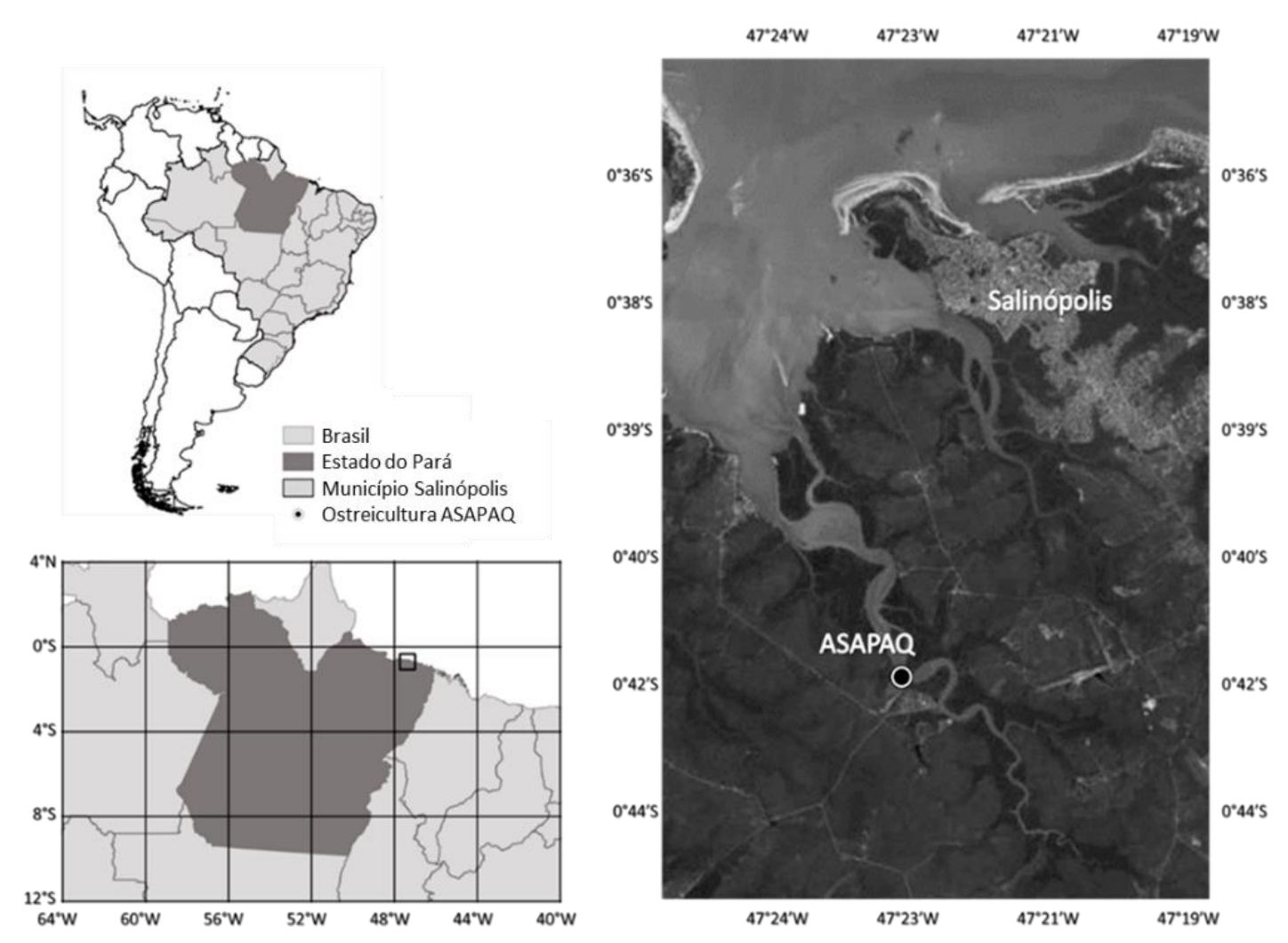

Figura 1: Localização do cultivo de ostras da Associação dos Agricultores, Pecuaristas e Aquicultores ASAPAQ, situado na zona estuarina da bacia hidrográfica do rio Urindeua, Pará.

Na ASAPAQ cultiva-se a ostra-do-mangue C. tulipa (Figura 2A) - espécie identificada a partir de análise genética dos exemplares em banco natural $[8,9,10,11,12]$ - comprando as sementes na Associação de Aquicultores de Vila de Lauro Sodré - AQUAVILA, município de Curuçá - PA $[13,14]$. Os membros da ASAPAQ adquirem entre 10 a 50 mil sementes de ostras por ano, que são dispostas em apetrechos (lanternas, bolsas e/ou travesseiros) de acordo com o estágio de desenvolvimento da ostra, utilizando a malha presente no apetrecho como seletor de tamanho. De acordo com os ostreicultores, as ostras atingem tamanho comercial $(>60 \mathrm{~mm})$ após aproximadamente seis meses e a produção de cada ostreicultor varia entre 10 a 15 mil unidades.

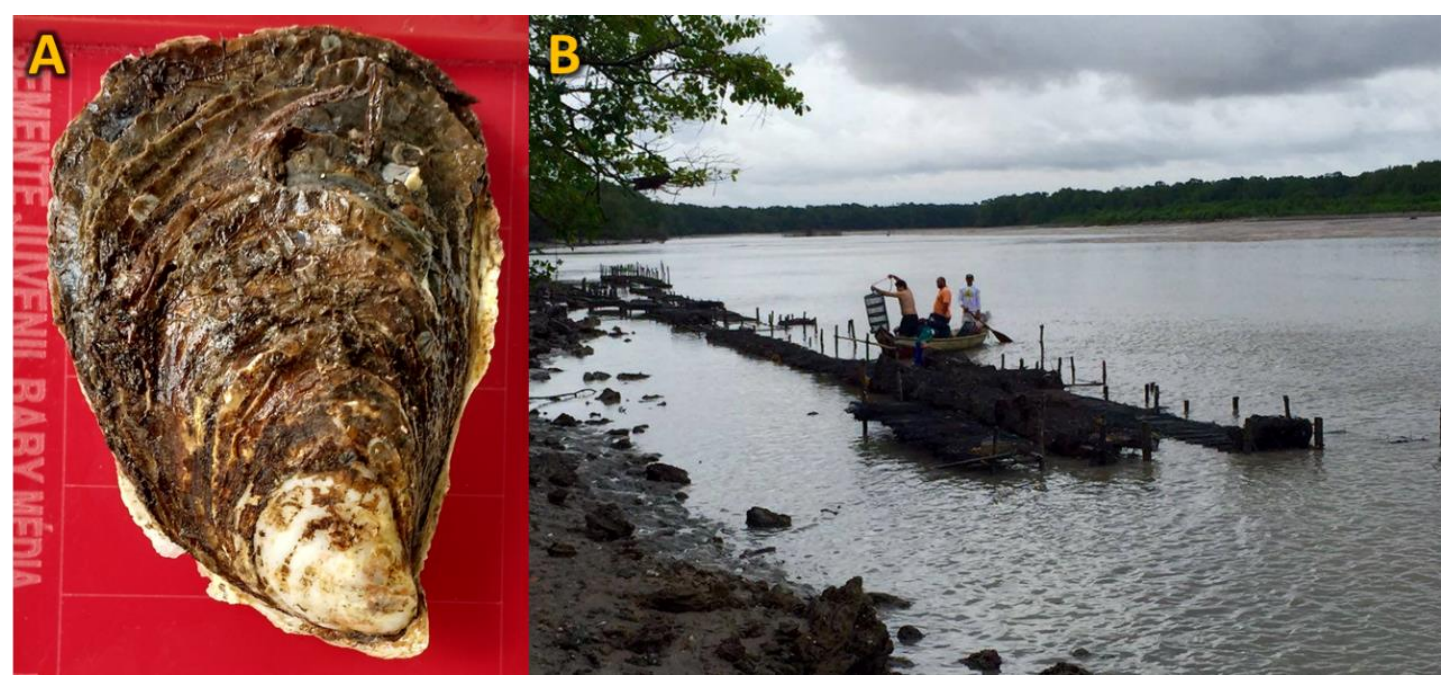

Figura 2: Ostra Crassostrea tulipa (A) cultivada na Associação dos Agricultores, Pecuaristase Aquicultores - ASAPAQ (B), no rio Urindeua. 


\subsection{Metodologia de amostragem}

Utilizou-se neste estudo um total de 1.028 ostras, adquiridas na ASAPAQ no mês de abril de 2016. Buscando uma maior amplitude nas classes dos indivíduos amostrados, utilizaram-se cinco tamanhos disponíveis no cultivo: "Semente" (15 a 29 mm de comprimento), "Juvenil" (30 a 59 $\mathrm{mm})$, "Baby" (60 a $79 \mathrm{~mm})$, "Média" (80 a $100 \mathrm{~mm}$ ), e "Master" (>100 mm), separadas empiricamente pelos ostreicultores. Paralelamente aos dados biométricos, mensurou-se a salinidade e a temperatura superficial da água no instante da coleta das ostras no cultivo, com um refratômetro manual e termômetro de imersão, respectivamente.

\subsection{Caracterização biomorfométrica}

A caracterizaçãobiométrica ocorreu com a determinação do comprimento total, largura e altura (em mm), bem como biomassa (g) das ostras coletadas. A morfometria dos indivíduos efetuou-se segundo Quayle (1988) [22], mensuradas com um paquímetro digital (TESA Data-Direct, precisão: $0,01 \mathrm{~mm}$ ) e a biomassa total úmida (concha + partes moles do bivalve + líquido intervalvar) determinou-se em balança digital (MH-Series Pocket Scale, precisão: 0,01 g). Devido a presença do líquido intervalvar, efetuou-se também a relação peso/comprimento da espécie considerando apenas a biomassa visceral da ostra, obtida após o desconchamento dos bivalves. $\mathrm{O}$ rendimento da carne comestível foi determinado através da relação biomassa visceral/biomassa total da ostra, utilizando indivíduos de todos os tamanhos.

\subsection{Análise dos dados}

Para descrever os aspectos morfométricos de C. tulipa, seguiu-se a metodologia utilizada por Gaspar et al. (2001) [23] com bivalves. Deste modo, efetuou-se relações morfométricas entre as medidas externas (comprimento total, largura e altura) da concha das ostras utilizando equações lineares, descrita na equação 1 abaixo:

$$
Y=a+b . X
$$

Para a relação peso/comprimento da espécie, efetuou-se relações entre as medidas externas da concha com as biomassas total e visceral, utilizando o modelo geométrico descrito na equação 2 abaixo:

$$
Y=a \cdot X^{b}
$$

Utilizou-se o coeficiente de correlação de Pearson $(r)$ para testar a existência de dependência estatística entre as variáveis das relações morfométricas e de peso/comprimento. Utilizou-se um test $t$ para verificar a significância dos valores de $r$ conforme Rodrigues (2010) [24]. Utilizou-se análise de variância (ANOVA, $\alpha=0,05$ ) para verificar possíveis diferenças entre os rendimentos da massa visceral dos tamanhos de ostras disponíveis.

Estimou-se os coeficientes linear $(a)$ e angular $(b)$ das regressões pelo método dos mínimos quadrados, sendo que para o modelo geométrico, utilizou-se a fórmula logarítmizada descrita na equação 3 abaixo:

$$
\ln Y^{\prime}=\ln a+b \cdot \ln X
$$

Posteriormente, classificou-se as equações conforme os valores do coeficiente angular $(b)$ de acordo com Vasconcelos e Gaspar (2017) [25]. Segundo os autores, as equações que utilizam o mesmo tipo de variável, a isometria ocorre quando $b=1$, enquanto que em relações entre diferentes tipos de variáveis a isometria ocorre quando $b=3$. Deste modo, equações que apresentam valores de $b$ superiores são classificadas por alometria positiva e aquelas que apresentam valores de $b$ inferiores são classificadas por alometria negativa. 


\subsection{Determinação do Indicador de Estabilização da Forma da concha (IEF)}

Para determinar o tamanho no qual C. tulipa atinge a forma definida da concha aplicou-se o método proposto por Gil et al. (2007) [26], que se denomina Indicador de Estabilização da Forma (IEF) da concha. Este método constitui-se por uma análise simples e consiste em calcular as razões, expressas em porcentagem, entre as três medidas lineares da concha. Para tanto, separouse os exemplares amostrados em classes de comprimento, calculadas através da regra da raiz quadrada ( $k=\sqrt{n}$, onde $k$ é o número de classes e $n$ é o número de ostras) e o intervalo de classes calculado pela equação $I C=(L s-L i) / k$, onde $I C$ é o intervalo de classe e $L s$ e $L i$ são os maiores e menores valores de comprimento total amostrado. Calculou-se a média das medidas lineares para cada intervalo, determinando-se as seguintes razões morfométricas: $A / C, L / C$ e $A / L$, sendo $A$ a altura, $C$ o comprimento total e $L$ a largura da concha.

Todas as análises foram realizadas a um nível de significância de 95\%, no programa PAST Palaeontological statistics (Versão 3.20).

\section{RESULTADOS}

Os dados brutos da biomorfometria de C. tulipa estão disponíveis em Chagas et al. (2016) [27], na plataforma digital Data Publisher for Earth \& Environmental Science - PANGAEA (www.pangaea.de). Os indivíduos coletados apresentaram comprimento total de 58,8 $\pm 25,7 \mathrm{~mm}$ (média $\pm \mathrm{SD}$ ), variando entre 16,0 e 116,9 mm, largura de 42,9 $\pm 19,0 \mathrm{~mm}$, medindo entre 10,6 e $82,2 \mathrm{~mm}$ e altura de $23,7 \pm 13,5 \mathrm{~mm}$, variando entre 2,97 e $77,82 \mathrm{~mm}$.

A relação comprimento e largura da concha, quando analisado por classes de tamanho comercial, de acordo com os valores de $r$, foram classificadas por correlações "substanciais" $(0,41>r<0,70)$, exceto a relação entre as ostras classificadas por "Master", com correlações "nulas" $(0>r<0,20)$ (Tabela 1$)$.

Os resultados das relações efetuadas entre o comprimento e a altura da concha de C. tulipa foram distintos entre as classes de comprimento, sendo a correlação entre "Semente" e "Baby" classificadas por correlação "fraca" $(0,21>r<0,40)$, entre tamanho "Juvenil" por "substanciais", entre "Média" e "Master" por correlações "nulas" (Tabela 1). A correlação entre as variáveis, sem distinção de classes, classificou-se por "extremamente fortes", sendo superior a encontrada em outros locais do Brasil (Tabela 2).

Os valores de $r$ apresentaram-se bem divergentes quando se relacionam as relações entre as medidas morfométricas com as biomassas totais e viscerais. Entretanto, destacam-se as relações da biomassa total com as medidas de comprimento e largura, classificadas por uma alometria negativa e positiva, respectivamente, porém com uma forte tendência a isometria $(b=3)$.

Os indivíduos apresentaram biomassa total de $62,3 \pm 54,8 \mathrm{~g}$, variando entre 0,6 e 101,8 g e biomassa visceral de $6,14 \pm 4,24 \mathrm{~g}$, entre 0,1 e $15,52 \mathrm{~g}$. Os rendimentos da biomassa visceral dos indivíduos analisados apresentaram média de 20,2 \%, com variação entre 7 e 44,7\%. Deste modo, calcula-se que, para adquirirmos $1 \mathrm{~kg}$ de carne de ostra sem conchas, deve-se adquirir aproximadamente $5 \mathrm{~kg}$ de ostras inteiras. A ANOVA evidenciou diferença do rendimento da carne da ostra entre as classes de comprimento ( $\mathrm{F}=3,99$ e $p<0,01)$, sendo evidente entre as classificadas por "Semente" com aquelas classificadas por "Baby" e "Master" (Figura 3). 
Tabela 1: Dados da dispersão bivariada entre comprimento total e largura da concha de Crassostrea tulipa com o total de indivíduos utilizados na relação $(\mathrm{N})$, a equação da relação, os coeficientes de correlação $\left(\mathrm{R}^{2}\right)$, coeficientes de correlação de Pearson $(\mathrm{r})$ e os valores de $\mathrm{p}$, sendo * valores menores que 0,001. Legenda: comprimento (C), largura (L), altura (A), biomassa total (Bt) e biomassa visceral (Bv).

\begin{tabular}{|c|c|c|c|c|c|c|c|}
\hline Classe & Relação & $N$ & Equação & $\boldsymbol{R}^{2}$ & $r$ & Alometria & $p$ \\
\hline \multirow{9}{*}{ "Semente" } & $C \times L$ & 234 & $L=1,33+0,48 C$ & 0,21 & 0,47 & - & $*$ \\
\hline & $C \times A$ & 234 & $A=-0,85+0,84 C$ & 0,14 & 0,38 & - & $*$ \\
\hline & $L \times A$ & 234 & $A=-0,11+0,66 L$ & 0,09 & 0,31 & - & $*$ \\
\hline & $C \times B t$ & 234 & $B t=-4,50+1,74 C$ & 0,43 & 0,66 & - & $*$ \\
\hline & $L \times B t$ & 234 & $B t=-2,83+1,32 L$ & 0.26 & 0,51 & - & $*$ \\
\hline & $A \times B t$ & 234 & $B t=-0,22+0,64 A$ & 0,28 & 0,53 & - & $*$ \\
\hline & $C \times B v$ & 8 & $B v=-12,17+3,68 C$ & 0,55 & 0,78 & + & 0,019 \\
\hline & $L \times B v$ & 8 & $B v=-9,51+2,82 L$ & 0,01 & 0,39 & - & 0,330 \\
\hline & $A \times B v$ & 8 & $B v=-4,39+2,06 A$ & 0,96 & 0,98 & - & $*$ \\
\hline \multirow{9}{*}{ "Juvenil" } & $C \times L$ & 209 & $L=0,59+0,73 C$ & 0,34 & 0,59 & - & $*$ \\
\hline & $C \times A$ & 209 & $A=-1,15+0,96 C$ & 0,22 & 0,47 & - & $*$ \\
\hline & $L \times A$ & 209 & $A=-0,92+1,00 L$ & 0,37 & 0,61 & $\mathrm{i}$ & $*$ \\
\hline & $C \times B t$ & 209 & $B t=-6,72+2,40 C$ & 0,46 & 0,68 & - & $*$ \\
\hline & $L \times B t$ & 209 & $B t=-4,81+2,10 L$ & 0,54 & 0,74 & - & $*$ \\
\hline & $A \times B t$ & 209 & $B t=-0,90+1,27 A$ & 0,53 & 0,73 & - & $*$ \\
\hline & $C \times B v$ & 38 & $B v=-9,17+2,61 C$ & 0,45 & 0,68 & - & $*$ \\
\hline & $L \times B v$ & 38 & $B v=-5,31+1,72 L$ & 0,36 & 0,61 & - & $*$ \\
\hline & $A \times B v$ & 38 & $B v=-2,34+1,15 A$ & 0,51 & 0,72 & - & $*$ \\
\hline \multirow{9}{*}{ "Baby" } & $C \times L$ & 320 & $L=0,28+0,86 C$ & 0,40 & 0,63 & - & $*$ \\
\hline & $C \times A$ & 320 & $A=0,87+0,60 C$ & 0,09 & 0,31 & - & $*$ \\
\hline & $L \times A$ & 320 & $A=1,87+0,40 L$ & 0,07 & 0,28 & - & $*$ \\
\hline & $C \times B t$ & 320 & $B t=-4,63+2,12 C$ & 0,47 & 0,69 & - & $*$ \\
\hline & $L \times B t$ & 320 & $B t=-1,28+1,43 L$ & 0,39 & 0,63 & - & $*$ \\
\hline & $A \times B t$ & 320 & $B t=0,99+0,98 A$ & 0,37 & 0,61 & - & $*$ \\
\hline & $C \times B v$ & 23 & $B v=-11,24+3,12 C$ & 0,13 & 0,41 & + & 0,045 \\
\hline & $L \times B v$ & 23 & $B v=3,06-0,25 L$ & $-0,04$ & 0,06 & - & 0,773 \\
\hline & $A \times B v$ & 23 & $B v=-4,11+1,82 A$ & 0,42 & 0,66 & - & $*$ \\
\hline \multirow{9}{*}{ "Média" } & $C \times L$ & 248 & $L=1,40+0,61 C$ & 0,16 & 0,41 & - & $*$ \\
\hline & $C \times A$ & 248 & $A=2,13+0,32 C$ & 0,01 & 0,15 & - & 0,016 \\
\hline & $L \times A$ & 248 & $A=2,47+0,26 L$ & 0,03 & 0,18 & - & 0,003 \\
\hline & $C \times B t$ & 248 & $B t=-3,23+1,80 C$ & 0,31 & 0,55 & + & $*$ \\
\hline & $L \times B t$ & 248 & $B t=-0,32+1,24 L$ & 0,32 & 0,56 & + & $*$ \\
\hline & $A \times B t$ & 248 & $B t=1,44+0,93 A$ & 0,39 & 0,63 & - & $*$ \\
\hline & $C \times B v$ & 41 & $B v=1,24+0,38 C$ & $-0,02$ & 0,03 & - & 0,833 \\
\hline & $L \times B v$ & 41 & $B v=-2,15+1,22 L$ & 0,01 & 0,17 & + & 0,260 \\
\hline & $A \times B v$ & 41 & $B v=-2,82+1,60 A$ & 0,16 & 0,42 & + & 0,005 \\
\hline \multirow{9}{*}{ "Master" } & $C \times L$ & 14 & $L=44,68+0,24 C$ & $-0,04$ & 0,17 & - & 0,545 \\
\hline & $C \times A$ & 14 & $A=38,55-0,01 C$ & $-0,08$ & 0,01 & - & 0,990 \\
\hline & $L \times A$ & 14 & $A=1,35+0,53 L$ & 0,06 & 0,37 & - & 0,188 \\
\hline & $C \times B t$ & 14 & $B t=3,86-0,04 C$ & $-0,08$ & 0,01 & - & 0,955 \\
\hline & $L \times B t$ & 14 & $B t=-3,22+1,96 L$ & 0,65 & 0,82 & - & $*$ \\
\hline & $A \times B t$ & 14 & $B t=2,81+0,63 A$ & 0,07 & 0,38 & - & 0,172 \\
\hline & $C \times B v$ & 5 & $B v=-21,26+5,22 C$ & 0,45 & 0,76 & + & 0,128 \\
\hline & $L \times B v$ & 5 & $B v=-3,22+1,48 L$ & 0,36 & 0,72 & - & 0,168 \\
\hline & $A \times B v$ & 5 & $B v=2,82+0,06 A$ & $-0,33$ & 0,03 & - & 0,957 \\
\hline \multirow{9}{*}{ Geral } & $C \times L$ & 1025 & $L=0,46+0,71 C$ & 0,95 & 0,97 & - & $*$ \\
\hline & $C \times A$ & 1025 & $A=-2,58+1,39 C$ & 0,91 & 0,95 & + & $*$ \\
\hline & $L \times A$ & 1025 & $A=-2,18+1,40 L$ & 0,92 & 0,95 & + & $*$ \\
\hline & $C \times B t$ & 1025 & $B t=-8,55+2,99 C$ & 0,96 & 0,98 & - & $*$ \\
\hline & $L \times B t$ & 1025 & $B t=-7,66+3,02 L$ & 0,96 & 0,98 & + & $*$ \\
\hline & $A \times B t$ & 1025 & $B t=-2,68+2,05 A$ & 0,95 & 0,97 & - & $*$ \\
\hline & $C \times B v$ & 115 & $B v=-9,85+2,82 C$ & 0,82 & 0,90 & - & $*$ \\
\hline & $L \times B v$ & 115 & $B v=-9,91+3,03 L$ & 0,75 & 0,87 & + & $*$ \\
\hline & $A \times B v$ & 115 & $B v=-4,29+1,94 A$ & 0,83 & 0,91 & - & $*$ \\
\hline
\end{tabular}


Tabela 2: Relações biomorfométricas de ostras Crassostrea spp. com o total de ostras utilizadas no estudo $(\mathrm{N})$, a equação da relação, os coeficientes de correlação $\left(\mathrm{R}^{2}\right)$, coeficientes de correlação de Pearson (r). Legenda: comprimento (C), largura (L), altura (A), biomassa total (Bt) e biomassa visceral (Bv).

\begin{tabular}{|c|c|c|c|c|c|c|c|}
\hline Espécie & Local & Relação & $N$ & Equação & $\overline{R^{2}}$ & $r$ & Referência \\
\hline C. angulata & $\begin{array}{c}\text { Estuário Kuala Gigieng, } \\
\text { Indonésia }\end{array}$ & Bt $\times \mathrm{C}$ & 442 & $\mathrm{Bt}=0,21 \times \mathrm{C}^{1,48}$ & 0,62 & 078 & Octavina et al. [28] \\
\hline C. ariakensis & Baía Chesapeake, EUA & $\mathrm{C} \times \mathrm{L}$ & 936 & $\mathrm{~L}=1,27-12,89 \mathrm{C}$ & 0,85 & 0,92 & Harding [29] \\
\hline C. ariakensis & Baía Chesapeake, EUA & $\mathrm{C} \times \mathrm{L}$ & 226 & $\mathrm{~L}=0,54+33,35 \mathrm{C}$ & 0,69 & 0,83 & Harding [29] \\
\hline C. ariakensis & Baía Chesapeake, EUA & $\mathrm{C} \times \mathrm{L}$ & 337 & $\mathrm{~L}=0,66+25,15 \mathrm{C}$ & 0,56 & 0,74 & Harding [29] \\
\hline C. ariakensis & Baía Chesapeake, EUA & $\mathrm{C} \times \mathrm{L}$ & 399 & $\mathrm{~L}=0,67+0,45 \mathrm{C}$ & 0,43 & 0,65 & Harding [29] \\
\hline C. ariakensis & Baía Chesapeake, EUA & $\mathrm{C} \times \mathrm{L}$ & 176 & $\mathrm{~L}=0,48+22,65 \mathrm{C}$ & 0,47 & 0,68 & Harding [29] \\
\hline C. ariakensis & Baía Chesapeake, EUA & $\mathrm{C} \times \mathrm{L}$ & 186 & $\mathrm{~L}=0,55+17,25 \mathrm{C}$ & 0,55 & 0,74 & Harding [29] \\
\hline C. ariakensis & Baía Chesapeake, EUA & $\mathrm{C} \times \mathrm{A}$ & 936 & $\mathrm{~L}=0,31-0,12 \mathrm{C}$ & 0,77 & 0,87 & Harding [29] \\
\hline C. ariakensis & Baía Chesapeake, EUA & $\mathrm{C} \times \mathrm{A}$ & 226 & $\mathrm{~L}=0,47-6,64 \mathrm{C}$ & 0,72 & 0,84 & Harding [29] \\
\hline C. ariakensis & Baía Chesapeake, EUA & $\mathrm{C} \times \mathrm{A}$ & 337 & $\mathrm{~L}=0,30+18,53 \mathrm{C}$ & 0,29 & 0,53 & Harding [29] \\
\hline C. ariakensis & Baía Chesapeake, EUA & $\mathrm{C} \times \mathrm{A}$ & 399 & $\mathrm{~L}=0,15+10,97 \mathrm{C}$ & 0,17 & 0,41 & Harding [29] \\
\hline C. ariakensis & Baía Chesapeake, EUA & $\mathrm{C} \times \mathrm{A}$ & 176 & $\mathrm{~L}=0,44-6,23 \mathrm{C}$ & 0,59 & 0,76 & Harding [29] \\
\hline C. ariakensis & Baía Chesapeake, EUA & $\mathrm{C} \times \mathrm{A}$ & 186 & $\mathrm{~L}=0,33+5,10 \mathrm{C}$ & 0,57 & 0,75 & Harding [29] \\
\hline C. brasiliana & Bertioga - SP, Brasil & $\mathrm{C} \times \mathrm{A}$ & 202 & $\mathrm{~A}=9,46+1,02 \mathrm{C}$ & 0,64 & 0,80 & Pereira et al. [30] \\
\hline C. brasiliana & Bertioga-SP, Brasil & $\mathrm{C} \times \mathrm{A}$ & 215 & $\mathrm{~A}=11,01+0,98 \mathrm{C}$ & 0,56 & 0,75 & Pereira et al. [30] \\
\hline C. brasiliana & Bertioga - SP, Brasil & $\mathrm{Bv} \times \mathrm{C}$ & 86 & $\mathrm{Bv}=3,2 \times 10^{-3} \times \mathrm{C}^{2, \mathrm{us}}$ & 0,65 & 0,80 & Pereira et al. [30] \\
\hline C. brasiliana & Bertioga - SP, Brasil & $\mathrm{Bv} \times \mathrm{A}$ & 88 & $\mathrm{Bv}=2,7 \times 10^{-4} \mathrm{xA}^{2,58}$ & 0,71 & 0,84 & Pereira et al. [30] \\
\hline C. brasiliana & Cananéia-SP, Brasil & $\mathrm{Bv} \times \mathrm{C}$ & 126 & $\mathrm{Bv}=-2,83+2,66 \mathrm{C}$ & - & - & Pereira \& Chagas Soares [31] \\
\hline C. brasiliana & Cananéia-SP, Brasil & Bt $\times \mathrm{C}$ & 126 & $\mathrm{Bt}=-1,32+2,71 \mathrm{C}$ & - & - & Pereira \& Chagas Soares [31] \\
\hline C. gigas & $\begin{array}{l}\text { Estuário Kuala Gigieng, } \\
\text { Indonésia }\end{array}$ & Bt $\times \mathrm{C}$ & 446 & $\mathrm{Bt}=4,5 \times 10^{-2} \times \mathrm{C}^{1,86}$ & 0,77 & 0,87 & Octavina et al. [28] \\
\hline C. gryphoides & Ilha Chorao,Índia & Bt $\times \mathrm{C}$ & 82 & $\mathrm{Bt}=-1,24+1,46 \mathrm{C}$ & 0,39 & 0,63 & Nagi et al. [32] \\
\hline C. gryphoides & Ilha Chorao,Índia & Bt $\times$ L & 82 & $\mathrm{Bt}=-0,73+1,24 \mathrm{~L}$ & 0,30 & 0,55 & Nagi et al. [32] \\
\hline C. gryphoides & Ilha Chorao,Índia & Bt $\times \mathrm{A}$ & 82 & $\mathrm{Bt}=-0,67+1,34 \mathrm{~A}$ & 0,50 & 0,71 & Nagi et al. [32] \\
\hline C. gryphoides & Ilha Chorao, Índia & $\mathrm{Bv} \times \mathrm{C}$ & 82 & $\mathrm{Bv}=-2,01+1,24 \mathrm{C}$ & 0,20 & 0,45 & Nagi et al. [32] \\
\hline C. gryphoides & Ilha Chorao, Índia & $B v \times L$ & 82 & $\mathrm{Bv}=-1,15+0,76 \mathrm{~L}$ & 0,07 & 0,28 & Nagi et al. [32] \\
\hline C. gryphoides & Ilha Chorao,Índia & $\mathrm{Bv} \times \mathrm{A}$ & 82 & $\mathrm{Bv}=-1,27+0,94 \mathrm{~A}$ & 0,17 & 0,42 & Nagi et al. [32] \\
\hline C. madrasensis & $\begin{array}{c}\text { Canal Moheskhali, } \\
\text { Bangladesh }\end{array}$ & Bt $\times$ C & 1470 & $\mathrm{Bt}=0,305 \times \mathrm{C}^{2,70}$ & 0,99 & 0,99 & Amin et al. [33] \\
\hline C. madrasensis & Ilha Chorao,Índia & $\mathrm{x} C$ & 219 & $\mathrm{Bt}=-2,22+2,06 \mathrm{C}$ & 0,60 & 0,78 & Nagi et al. [32] \\
\hline C. madrasensis & Ilha Chorao, Índia & Bt $\times$ L & 219 & $\mathrm{Bt}=-1,15+1,53 \mathrm{~L}$ & 0,44 & 0,67 & Nagi et al. [32] \\
\hline C. madrasensis & Ilha Chorao, Índia & Bt $\times \mathrm{A}$ & 219 & $\mathrm{Bt}=-0,80+1,41 \mathrm{~A}$ & 0,47 & 0,69 & Nagi et al. [32] \\
\hline C. madrasensis & Ilha Chorao,Índia & $\mathrm{Bv} \times \mathrm{C}$ & 219 & $\mathrm{Bv}=-3,29+2,07 \mathrm{C}$ & 0,40 & 0,64 & Nagi et al. [32] \\
\hline C. madrasensis & Ilha Chorao,Índia & Bv $x \mathrm{~L}$ & 219 & $\mathrm{Bv}=-1,99+1,39 \mathrm{~L}$ & 0,25 & 0,50 & Nagi et al. [32] \\
\hline C. madrasensis & Ilha Chorao,Índia & $\mathrm{Bv} \times \mathrm{A}$ & 219 & $\mathrm{Bv}=-1,94+1,48 \mathrm{~A}$ & 0,36 & 0,60 & Nagi et al. [32] \\
\hline C. madrasensis & Nerul Creek, Índia & Bt $\times C$ & 203 & $\mathrm{Bt}=-1,64+1,72 \mathrm{C}$ & 0,51 & 0,72 & Nagi et al. [32] \\
\hline C. madrasensis & Nerul Creek, Índia & Bt $\times$ L & 203 & $\mathrm{Bt}=-0,79+1,29 \mathrm{~L}$ & 0,36 & 0,60 & Nagi et al. [32] \\
\hline C. madrasensis & Nerul Creek, Índia & Bt $\times \mathrm{A}$ & 203 & $\mathrm{Bt}=-0,44+1,17 \mathrm{~A}$ & 0,34 & 0,59 & Nagi et al. [32] \\
\hline C. madrasensis & Nerul Creek, Índia & $\mathrm{Bv} \times \mathrm{C}$ & 203 & $\mathrm{Bv}=-2,77+1,70 \mathrm{C}$ & 0,24 & 0,49 & Nagi et al. [32] \\
\hline C. madrasensis & Nerul Creek, Índia & $\mathrm{Bv} \times \mathrm{L}$ & 203 & $\mathrm{Bv}=-1,60+1,06 \mathrm{~L}$ & 0,10 & 0,33 & Nagi et al. [32] \\
\hline C. madrasensis & Nerul Creek, Índia & $\mathrm{Bv} \times \mathrm{A}$ & 203 & $\mathrm{Bv}=-1,69+1,24 \mathrm{~A}$ & 0,18 & 0,43 & Nagi et al. [32] \\
\hline C. madrasensis & Nerul Creek, Índia & Bt $\times \mathrm{C}$ & 115 & $\mathrm{Bt}=-2,11+1,99 \mathrm{C}$ & 0,54 & 0,74 & Nagi et al. [32] \\
\hline C. madrasensis & Nerul Creek, Índia & Bt $\times$ L & 115 & $\mathrm{Bt}=-1,18+1,52 \mathrm{~L}$ & 0,40 & 0,64 & Nagi et al. [32] \\
\hline C. madrasensis & Nerul Creek, Índia & Bt $\times \mathrm{A}$ & 115 & $\mathrm{Bt}=-0,64+1,29 \mathrm{~A}$ & 0,43 & 0,66 & Nagi et al. [32] \\
\hline C. madrasensis & Nerul Creek, Índia & $\mathrm{Bv} \times \mathrm{C}$ & 115 & $\mathrm{Bv}=-2,12+1,23 \mathrm{C}$ & 0,16 & 0,41 & Nagi et al. [32] \\
\hline C. madrasensis & Nerul Creek, Índia & Bv $x \mathrm{~L}$ & 115 & $\mathrm{Bv}=-2,01+1,27 \mathrm{~L}$ & 0,23 & 0,48 & Nagi et al. [32] \\
\hline C. madrasensis & Nerul Creek, Índia & $\mathrm{Bv} \times \mathrm{A}$ & 115 & $\mathrm{Bv}=-1,37+0,93 \mathrm{~A}$ & 0,18 & 0,43 & Nagi et al. [32] \\
\hline C. rhizophorae & Ilha de Margarita, Venezuela & Bt $\times \mathrm{C}$ & & $\mathrm{Bt}=3,3 \times 10^{-5} \times \mathrm{C}^{1,46}$ & 0,43 & 0,66 & Gonzalez et al. [34] \\
\hline C. iridescens & $\begin{array}{l}\text { Estuário Kuala Gigieng, } \\
\text { Indonésia }\end{array}$ & Bt $\times \mathrm{C}$ & 286 & $\mathrm{Bt}=0,40 \times \mathrm{C}^{1,27}$ & 0,71 & 0,84 & Octavina et al. [28] \\
\hline C. virginica & Baía Chesapeake, EUA & Bt $\times \mathrm{C}$ & - & $\mathrm{Bt}=\underset{\mathrm{C}^{2,49}}{6,79 \times 10^{-5} \mathrm{x}}$ & 0,94 & 0,96 & Paynter \& Dimichele [35] \\
\hline C. virginica & Machona, México & Bt $\times \mathrm{C}$ & - & $\mathrm{Bt}=0,06 \times \mathrm{C}^{1, \supset 0}$ & 0,90 & 0,95 & Cárdenas \& Aranda [36] \\
\hline C. virginica & Cascajal, México & Bt $\times \mathrm{C}$ & - & $\mathrm{Bt}=0,04 \times \mathrm{C}^{2,13}$ & 0,60 & 0,78 & Cárdenas \& Aranda [36] \\
\hline C. virginica & Puente de Ostión, México & Bt $\times \mathrm{C}$ & - & $\mathrm{Bt}=0,01 \times \mathrm{C}^{2,03}$ & 0,39 & 0,63 & Cárdenas \& Aranda [36] \\
\hline C. virginica & Pama I, México & Bt $\times \mathrm{C}$ & - & $\mathrm{Bt}=0,01 \times \mathrm{C}^{2,01}$ & 0,49 & 0,70 & Cárdenas \& Aranda [36] \\
\hline C. virginica & Pama II, México & Bt $\times \mathrm{C}$ & - & $\mathrm{Bt}=0,62 \times \mathrm{C}^{0,92}$ & 0,21 & 0,46 & Cárdenas \& Aranda [36] \\
\hline C. virginica & Baía Chesapeake, EUA & $\mathrm{C} \times \mathrm{L}$ & 399 & $\mathrm{~L}=0,63+2,71 \mathrm{C}$ & 0,51 & 0,71 & Harding [29] \\
\hline C. virginica & Baía Chesapeake, EUA & $\mathrm{C} \times \mathrm{L}$ & 168 & $\mathrm{~L}=0,50+18,82 \mathrm{C}$ & 0,53 & 0,72 & Harding [29] \\
\hline C. virginica & Baía Chesapeake, EUA & $\mathrm{C} \times \mathrm{L}$ & 166 & $\mathrm{~L}=0,45+26,05 \mathrm{C}$ & 0,36 & 0,60 & Harding [29] \\
\hline C. virginica & Baía Chesapeake, EUA & $\mathrm{C} \times \mathrm{A}$ & 399 & $\mathrm{~L}=0,18+5,30 \mathrm{C}$ & 0,36 & 0,60 & Harding [29] \\
\hline C. virginica & Baía Chesapeake, EUA & $\mathrm{C} \times \mathrm{A}$ & 168 & $\mathrm{~L}=0,38-3,10 \mathrm{C}$ & 0,60 & 0,77 & Harding [29] \\
\hline C. virginica & Baía Chesapeake, EUA & $\mathrm{C} \times \mathrm{A}$ & 166 & $\mathrm{~L}=0,23+10,75 \mathrm{C}$ & 0,32 & 0,56 & Harding [29] \\
\hline C. virginica & Baía Chesapeake, EUA & Bt $\times \mathrm{C}$ & - & $\mathrm{Bt}=3 \mathrm{e}-05 \times \mathrm{C}^{2,30}$ & 0,73 & 0,85 & Liddel [37] \\
\hline C. virginica & New Hampshire, USA & Bt $\times \mathrm{C}$ & - & $\mathrm{Bt}=1,8 \times 10^{-6} \times \mathrm{C}^{3,37}$ & 0,92 & 0,95 & Grizzle et al. [38] \\
\hline C. virginica & $\begin{array}{l}\text { Estuário Kuala Gigieng, } \\
\text { Indonésia }\end{array}$ & Bt $\times \mathrm{C}$ & 377 & $\mathrm{Bt}=5,4 \times 10^{-2} \times \mathrm{C}^{1,86}$ & 0,70 & 0,83 & Octavina et al. [28] \\
\hline
\end{tabular}




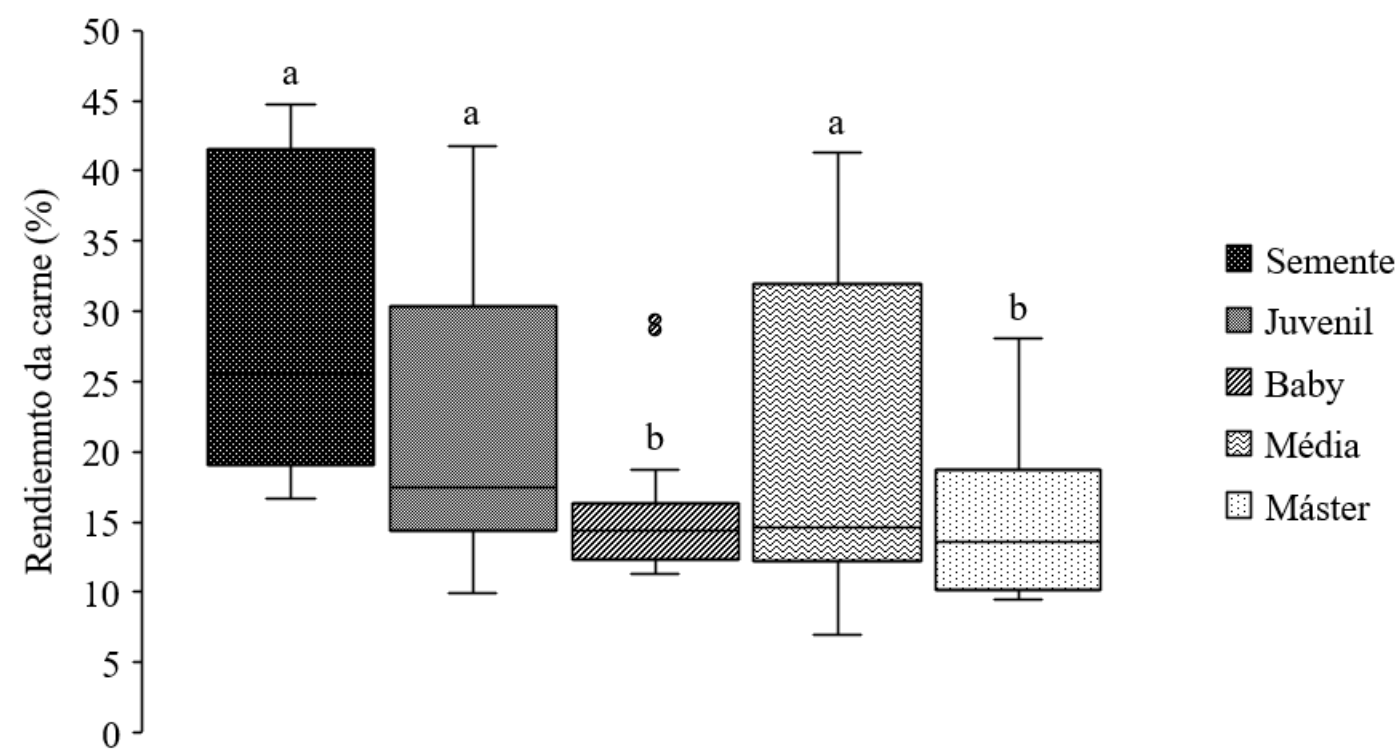

Figura 3: Rendimento percentual médio da carne (massa visceral) da ostra Crassostrea tulipa cultivada no litoral amazônico, Pará.

Os resultados na análise de IEF remete a uma oscilação entre as medidas morfométricas ao longo do desenvolvimento de C. tulipa, porém observa-se uma tendência a estabilização a partir de $60 \mathrm{~mm}$ (Figura 4). Evidencia-se que a maior variaçãona forma da concha dos indivíduos ocorre nos tamanhos "Semente" e "Juvenil" e que a estabilização coincide com o tamanho comercial da ostra cultivada. Esta foi a primeira vez que se utilizou a análise de IEF para ostras do gênero Crassostrea. Tais resultados são importantes pois, a nível comercial, a "aparência" externa da concha é primordial na decisão de aquisição pelos consumidores.

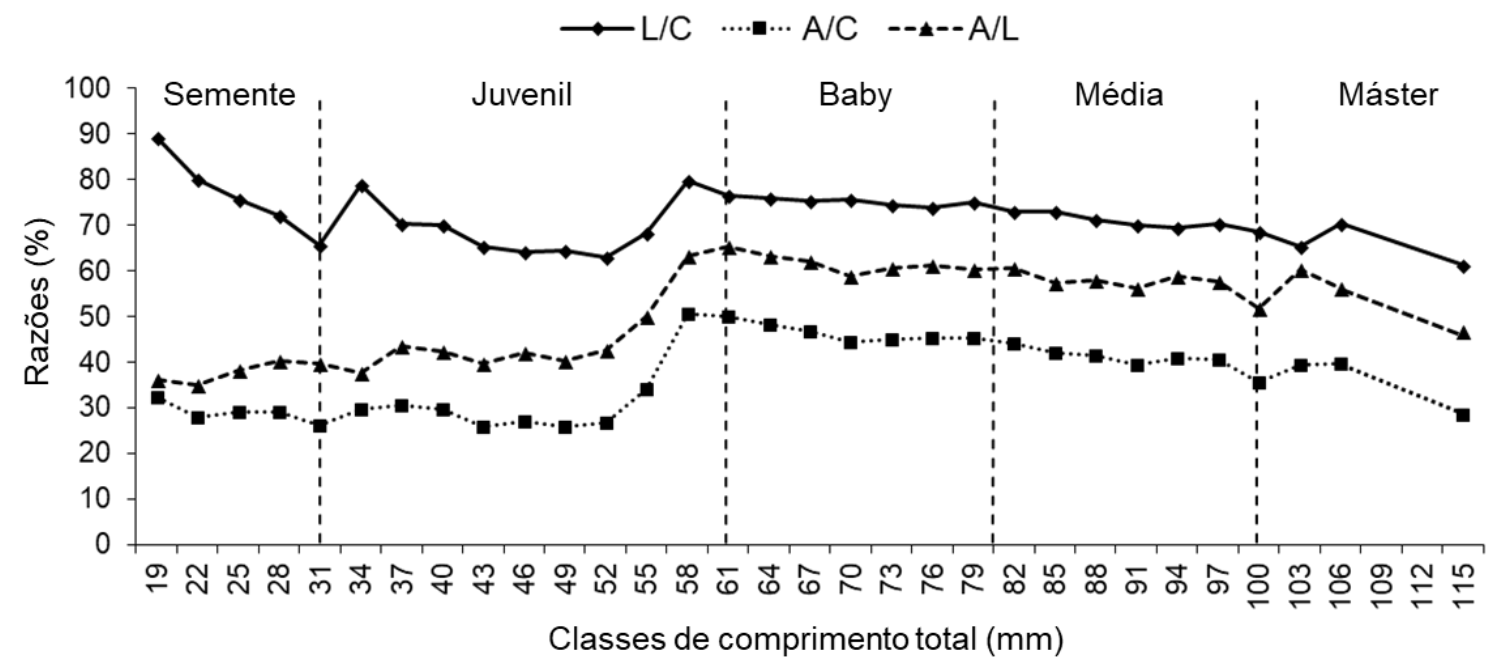

Figura 4: Representação gráfica do Índice de Estabilização da Forma (IEF) da concha de Crassostrea tulipa no Pará. Legenda: comprimento $(C)$, largura $(L)$ e altura $(A)$.

\section{DISCUSSÃO}

Estudos biomorfométricos em bivalves apresentam equações que inferem a determinação de variáveis (biomassa e morfometria) importantes à gestão do recurso pesqueiro, principalmente de espécies de interesse comercial [25]. Na ostreicultura, diversos autores abordam as relações biomorfométricas de ostras do gênero Crassostrea (ver Tabela 2). Essas contribuições favorecem 
o cultivo, pois aferem dados importantes (e.g., estimação morfométricos e rendimento da carne) ao manejo durante a produção.

Os resultados das relações entre o comprimento e largura são similares ao encontrado por Harding (2007) [29], em seu estudo com Crassotrea virginica e C. ariakensis (Tabela 2). Entretanto ao analisar a relação entre as variáveis de um modo geral (sem distinção de classes), este atual estudo apresentou resultados melhores do que encontrado por esse autor, evidenciando uma correlação classificada por "extremamente forte" $(0,91>r<1,0)$. Resultados das relações entre comprimento e altura também foram similares aos encontrados por Harding (2007) [29] e Pereira et al. (1988) [30].

Os valores de $r$ divergentes nas relações das medidas morfométricas com as biomassas totais e viscerais são similares aos valores encontrados por outros autores $[28,30,31,32,33,34,36$, 37, 38] (Tabela 2).

O rendimento da carne comestível neste estudo foi semelhante ao encontrado por Lenz (2008) [39] em C. tulipa cultivada na Baía de Camaru, no estado da Bahia, variando entre 8 a 17,7 \% em exemplares de 30,4 a 94,6 mm. Galvão et al. (2000) [40] observou que em C. brasiliana os maiores rendimentos de biomassa visceral de ostras são naquelas maduras sexualmente, devido a acumulação de reservas e do pleno desenvolvimento dos folículos. Considerando o resultado do atual trabalho e o verificado por Galvão et al. (2000) [40] com a ostra C. brasiliana, ressalta-se uma relação inversa ao rendimento encontrado por classes de tamanho. Sendo assim, enquanto esses autores evidenciaram maiores rendimentos da carne em indivíduos adultos, neste estudo, os maiores rendimentos foram encontrados na classe "Semente" (Figura 2).

Os rendimentos da biomassa visceral de C. tulipa apresentam-se baixos ao compararmos com outros recursos aquáticos, tais como, peixes que possuem de 30 a 50\% [41] e camarões em torno de 50\% [42]. Entretanto apresentaram-se superior ao rendimento de outros invertebrados, tais como, caranguejos de 11 a $18 \%$ [43] e bivalves como Anomalocardiaflexuosa (Linnaeus, 1767) com $10 \%$ [44] e Donax striatus Linnaeus, 1767 com 12\% [45].

Os resultados apresentamuma tendência a dimorfismo da espécie ao longo de seu crescimento, porém com uma tendência a estabilização da forma da concha a partir de $60 \mathrm{~mm}$, coincidindo com o tamanho comercial da espécie (>60 mm) segundo Nascimento et al. (1980) [18]. As elevadas variações apresentadas em "Semente", "Juvenil" e "Master", possivelmente, devem-se ao número de indivíduos amostrados. Essa diferença nas proporções entre as medidas de comprimento nos grupos iniciais de crescimento ("Semente" e "Juvenil") pode ser explicada devido ao crescimento acelerado das ostras nos primeiros meses de vida [46].

Os aspectos biomorfométricos das ostras (e.g., a relação peso-comprimento), sãoinfluenciados diretamente por diversos fatores, dentre eles, a espécie estudada, o ambiente (e.g., natural ou em cultivo) [47, 48]. Além dessas variáveis, há outras que são importantes de se analisar, tais como: fatores abióticos (temperatura e/ou salinidade) [17, 46, 49, 50, 51, 52, 53, 54], local a ser cultivado (ambiente marinho ou estuarino) [55, 56, 57], tipo de estrutura do cultivo [58, 59, 60, 61], densidade estocada [62, 63, 64, 65], ambientes impactados [66] e o biofouling [6,21, 67, 68, 69, 70]. De acordo com esses estudos, elevadas temperaturas incrementam a biomassa das ostras, devido a maior disponibilidade de nutrientes na água o que estar associado a um maior crescimento dos bivalves.

\section{CONCLUSÃO}

Crassotrea tulipa apresenta excelentes relações biomorfométricas, gerando equações que satisfazem estimação de medidas morfométricas da concha. O rendimento da carne comestívelde C. tulipa é em média 20,2\% da biomassa total, podendo ser calculada através da equação gerada com as medidas externas da concha (comprimento, largura e altura).

Com o conhecimento das relações biomorfométricas e da biomassa comestível de ostras cultivadas, ocorre um melhor aproveitamento e dinamização do manejo dos bivalves, podendo estimar, com exatidão, a quantidade de ostra consumidas a partir do conhecimento de uma das medidas externas da concha.

Através da análise de IEF, evidencia-se que as ostras apresentam variação na forma da concha ao longo de todo seu desenvolvimento. Entretanto, observa-se uma tendência a estabilização ao atingir $60 \mathrm{~mm}$, tamanho ideal à comercialização. Recomenda-se uma análise de IEF em ostras 
oriundas de ambientes naturais, para verificar o comportamento biomorfométrico, principalmente, durante as fases iniciais de crescimento (classes "Semente" e "Juvenil"). Paralelamente, recomenda-se também, a análise de IEF sob influência da densidade de ostras e tipo de estrutura no qual a ostra está sendo cultivada.

\section{AGRADECIMENTOS}

Os autores agradecem a Associação dos Agricultores, Pecuaristas e Aquicultores (ASAPAQ) da Vila de Santo Antônio de Urindeua, pelo apoio ao desenvolvimento da pesquisa. em especial aos ostreicultores: a Dona Maria (presidente em exercício), Tito, Miro e seu Antônio (expresidente) Ao Conselho Nacional de Desenvolvimento Científico Tecnológico (CNPq), pela concessãode bolsas para a realização desta pesquisa. A Universidade Federal Rural da Amazônia, sobretudo ao Instituto Socioambiental e dos Recursos Hídricos, pelo apoio logístico na cessão do transporte ao deslocamento dos autores ao local da pesquisa.

\section{REFERÊNCIAS BIBLIOGRÁFICAS}

1. Castilho-Westphal GG, Ostrensky A. Use of side-scan sonar for estimations of Crassostrea brasiliana (Lamarck, 1819) stocks in subtidalbanks on the south coast of Brazil. Braz J Oceanogr. 2016;64(1):4956. doi: 10.1590/S1679-87592016104506401

2. Beck MW, Brumbaugh RD, Airoldi L, Carranza A, Coe LD, Crawford C, Defeo O, Edgar GJ, Hancock B, Kay MC, Lenihan HS, Luckenbach MW, Toropova CL, Zhang G, Guo X. Oyster reefs at risk and recommendations for conservation, restoration, and management. BioScience. 2011;61(2):107-16. doi: 10.1525/bio.2011.61.2.5

3. Shumway SE, Davis C, Downey R, Karney R, Kraeuter J, Parsons J, Rheault R, Wikfors G. Shellfish aquaculture in praise of sustainable economies and environments. World aquaculture. 2003;34(4):1518. Dis ponível em: http://darc.cms.udel.edu/ibog/ShumwayW ASarticle.pdf

4. FAO. The state of world fisheries and aquaculture. Rome: FAO, 2016. 200 p.

5. IBGE. Produção da pecuária municipal 2016. Rio de Janeiro: IBGE, 2017. 51 p. Disponível em: https://sidra.ibge.gov.br/tabela/3940\#resultado

6. Chagas RA. Biofouling no cultivo da ostra-do-mangue Crassostrea rhizophorae (Guilding, 1828) (Bivalvia: Ostreidae) em um estuário amazônico [Monografia]. Belém, PA: Universidade Federal Rural da Amazônia, 2016. 116 p. doi: 10.2312/ebt.thesis .chagas.2016

7. Hernandez OD, Troccoli LG, Millán JQ. Crecimiento,engorde y sobrevivencia de la ostra de mangle Crassostrea rhizophorae Guilding, 1828 en la Isla de Cubagüa, Venezuela. Carib J Sci. 1998;34(34):243-49.

8. Baldez RSC, Melo MAD, Sampaio I, Tagliaro $\mathrm{CH}$. Novel microsatellite markers for brazilian mangrove oysters (Crassostrea gasar) and their cross-amplification in Crassostrea rhizophorae. Braz Arch Biol Technol. 2016;59:e16160071. doi:10.1590/1678-4324-2016160071

9. Melo MAD, da Silva ARB, Beasley CR, Tagliaro CH. Multiplex species-specific PCR identification of native and non-native oysters (Crassostrea) in Brazil: a useful tool for application in oyster culture and stock management. Aquac Internat. 2013;21(6):1325-32. doi:10.1007/s 10499-013-9635-8

10. Melo MD, Silva ARB, Varela ES, Sampaio I, Tagliaro CH. Development and characterization of ten microsatellite markers for population studies of the native Brazilian oyster Crassostrea gasar. Conserv Genet Resour. 2012;4:582-86. doi:10.1007/s 12686-011-9597-y

11. Melo AGC, Varela ES, Beasley CR, Schneider H, Sampaio I, Gaffney PM, Reece KS, Tagliaro CH. Molecular identification, phylogeny and geographic distribution of Brazilian mangrove oysters (Crassostrea). Genet Mol Biol. 2010;33(3):564-72.

12. Varela ES, Beasley CR, Schneider H, Sampaio I, Marques-Silva NS, Tagliaro CH. Molecular phylogeny of mangrove oyster (Crassostrea) from Brazil. J Molluscan Stud. 2007;73:229-34. doi:10.1093/mo llus/eym018

13. Macedo ARG, Silva FL, Ribeiro SCA, Torres MF, Silva FNL, Medeiros LR. Perfil da ostreicultura na comunidade de Santo Antônio do Urindeua, Salinópolis, nordeste do Pará/Brasil. Rev Observatorio Econ Latinoam. 2016. Marzo: 1-25. Disponível em: http://www.eumed.net/cursecon/ecolat/br/16/aquicultura.html

14. Sampaio DS, Tagliaro CH, Schneider H, Beasley CR. Oyster culture on the Amazon mangrove coast: asymmetries and advances in an emerging sector. Rev Aquac. 2017;0:1-17. doi: 10.1111/raq.12227 
15. Hoshino P. Avaliação e comparação de projetos comunitários de ostreicultura localizados no nordeste paraense [Dis sertação]. Belém: Universidade Federal do Pará, 2009. 99 p.

16. Chagas RA, Herrmann M. Indução a desova de Crassostrea rhizophorae (Guilding, 1828) (Bivalvia: Ostreidae) através de métodos físico-químicos em condições controladas. Acta Fish Aquat Resour. 2015;3(2):24-30. doi:10.2312/ActaFish.2015.3.2.24-30

17. Funo ICSA, Antonio ÍG, Marinho YF, Gálvez AO. Influência da salinidade sobre a sobrevivência e crescimento de Crassostrea gasar. Boletim do Instituto de Pesca. 2015. 41(4): 837-47. Disponível em: https://www.pesca.sp.gov.br/41_4_837-847.pdf

18. Nascimento IA, Pereira SA, Souza RC. Determination of the optimum comercial size for the magrove oysters Crassostrea rhizophorae in Todos os Santos Bay, Brazil. Aquacult. 1980;20(1):1-8.

19. Chagas RA, Herrmann M. Estimativas de crescimento de bivalves tropicais e subtropicais: recomendação para um método padronizado. Acta Fish Aquat Resour. 2016;4(2):28-38. doi:10.2312/ActaFish.4016.4.2.28-38

20. Montanhini-Neto R, Ostrensky A. Revisão: Uso de modelos matemáticos para avaliação da influência de variáveis ambientais sobre o desenvolvimento de ostras no Brasil. PUBVET. 2012;6(4):1-33. Disponível em: http://bit.ly/2sCUhVT

21. Chagas RA, Barros MRF, Santos WCR, Herrmann M. Composition of the biofouling community associated with oyster culture in an Amazon estuary, Para state, Northern Brazil. Rev Biol Marina Oceanogr. 2018;53(1):9-17. doi:10.4067/S0718-19572018000100009

22. Quayle DB. Pacific oyster culture in British Columbia. Canadian Bulletin of Fisheries and Aquatic Sciences 218: 1988. 241 p. Disponível em: http://www.dfo-mpo.gc.ca/library/109165.pdf

23. Gaspar MB, Santos MN, Vasconcelos P. Weight-length relationships of 25 bivalve species (Mollusca: Bivalvia) from the Algarve coast (southern Portugal). J Mar Biol Assoc U.K. 2001;81:805-07. doi:10.1017/S0025315401004623

24. Rodrigues WC. Estatística Aplicada. Paracambi: W.C. Rodrigues, 2010. 70 p.

25. Vasconcelos P, Gaspar M. A importância e utilidade dos estudos morfométricos e do crescimento relativo em bivalves e gastrópodes. Portugala. 2017. 20: 10-11. Disponível em: https://is suu.com/portugala/docs/portugala20

26. Gil GM, Troncoso JS, Thomé JW. Shell shape stabilization indicator (IEF): Handling and optimization of bivalves mollusks exploitation. Porto Alegre: Edição do autor, 2007. 152 p.

27. Chagas RA, Barros MRF, Assis AS, Silva REO, Passos TAF, Santos WCR, Herrmann M. Morphometry and biomass of the cultured mangrove oyster Crassostrea rhizophorae at the Urindeua river, eastern Amazonia, northern Brazil. PANGAEA. 2016, doi:10.1594/PA NGAEA.864155

28. Octavina C, Yulianda F, Krisanti M, Muchlisin ZA. Length-weight relationship of Ostreidae in the Kuala Gigieng estuary, Aceh Besar District, Indonesia. Int J Bioflux Soc. 2015;8(5):817-23. Disponível em: http://www.bioflux.com.ro/docs/2015.817-823.pdf

29. Harding JM. Comparis on of growth rates between diploid deby eastern oysters (Crassostrea virginica, Gmelin 1791), triploid eastern oysters, and triploid suminoe oysters (C. ariakensis, Fugita 1913). J Shellfish Res. 2007;26(4):961-72. doi:10.2983/0730-8000(2007)26[961:COGRBD]2.0.CO;2

30. Pereira OM, Akaboshi S, Chagas Soares F. Cultivo experimental de Crassostrea brasiliana (Lamarck,

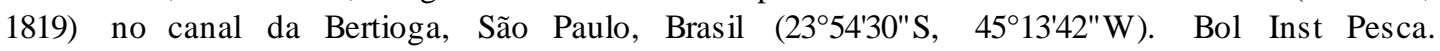
1988;15(1):55-65. Disponível em: https://www.pesca.sp.gov.br/15_1_55-65.pdf

31. Pereira OM, Chagas Soares Fd. Análise da criação de ostra Crassostrea brasiliana (Lamarck, 1819), no sítio Guarapari, na região lagunar-estuarina de Cananéia-SP. Bol Inst Pesca. 1996; 23(único):13542. Disponível em: https://www.pesca.sp.gov.br/23_135-142.pdf

32. Nagi HM, Shenai-Tirodkar PS, Jagtap G. Dimensional relationships in Crassostrea madrasensis (Preston) and $C$. gryphoides (Schlotheim) in mangrove ecosystem.Indian J Geo-Marine Sci. 2011;40(4):559-66. Disponível em: http://drs.nio.org/drs/handle/2264/3935

33. Amin SMN, Zafar M, Halim A. Age, growth, mortality and population structure of the oyster, Crassostrea madrasensis, in the Moheskhali Channel (southeastern coast of Bangladesh). J Appl Ichthyol. 2008;24(1):18-25. doi:10.1111/j.1439-0426.2007.01007.x

34. Gonzalez C, Crescini R, Villalba W, Maldonado A, Vásquez G, Soto G. Estructura de tallas, crecimiento y mortalidad de Crassostrea rhizophorae en la laguna de la Restinga, is la de Margarita, Venezuela. SABER. Rev Multid Consejo Invest Univ Oriente. 2015;27(2):328-33. Disponível em: http://www.redalyc.org/articulo.oa?id=427742245015

35. Paynter KT, Dimichele L. Growth of tray-cultured oyster (Crassostrea virginica Gmelin) in Chesapeake Bay. Aquac. 1990;87:289-97. 
36. Cárdenas ERB, Aranda DA. Differences in the exploited oyster (Crassostrea virginica (Gmelin, 1791)) populations from different coastal lagoons of the Gulf of Mexico. Trans1 Waters Bull. 2007;1(2):21-35. doi:10.1285/i1825229Xv1n2p21

37. Liddel MK. A von bertalanffy based model for the estimation of oyste (Crassostrea virginica) growth on restored oyster reefs in chesapeake bay [Tese]. University of Maryland, 2008. 171p.

38. Grizzle RE, Ward KM, Peter CR, Cantwell M, Katz D, Sullivan J. Growth, morphometrics and nutrient content of farmed eastern oysters, Crassostrea virginica (Gmelin), in New Hampshire, USA. Aquac Res. 2016;1-13. doi:10.1111/are.12988

39. Lenz TM. Biologia reprodutiva da ostra-do-mangue Crassostrea rhizophorae (Guilding, 1828) (Bivalvia: Ostreidae) como subsídio à implantação de ostreicultura na Baía de Camamu (BA) [Dis sertação]. Ilhéus - BA: Universidade Estadual de Santa Cruz, 2008. 54 p.

40. Galvão MSN, Pereira OM, Machado IC, Henriques MB. Aspectos reprodutivos da ostra Crassostrea brasiliana de manguezais do estuário de cananéia, SP $\left(25^{\circ} \mathrm{S} ; 48^{\circ} \mathrm{W}\right)$. Boletim de Instituto de Pesca. 2000. 26(2): 147-62. Dis ponível em: https://www.pesca.sp.gov.br/26_2_147-162.pdf

41. Macedo-Viegas EM, Souza MLR. Tópicos especiais em piscicultura de água doce tropical intensiva. São Paulo: Funep, 2004. Pré-processamento e conservação do pescado produzido em piscicultura, p. 405-80.

42. Lima SBP, Rabello CB-V, Dutra-Junior WM, Ludke MCMM, Costa FGP. Avaliação nutricional da farinha da cabeça de camarão marinho (Litopenaeus vannamei) para frangos de corte. Rev Caatinga. 2007;20(3):35-39.

43. Ogawa M, Silva AIM, Ogawa NBP, Mala EL, Nunes ML. Adequações tecnológicas no processamento da carne de caranguejo. Ci Tecnol Alim. 2008;28(1):78-82.

44. Chagas RA, Silva FBA, Vale AVP, Herrmann M. Morphometry and biomas of the bivalve Anomalocardia flexuosa at the beach Ajuruteua, State of Para, Brazil. PANGAEA. 2014. doi:10.1594/PANGAEA.830381.

45. Chagas RA, Silva FBA, Vale AVP, Herrmann M. Morphometry and biomass of the bivalve Donax striatus at the beach Ajuruteua, State of Pará, Brazil. PANGAEA. 2014. doi:10.1594/PANGAEA.830344.

46. Buitrago E, Buitrago J, Freites L, Lodeiros C. Identificación de factores que afectan al crecimiento y la supervivencia de la ostra de mangle, Crassostrea rhizophorae (Guilding, 1828), bajo condiciones de cultivo suspendido en la laguna de La Restinga, Isla de Margarita, Venezuela. Zootec Trop. 2009;27(1):79-90. Dis ponível em: http://www.scielo.org.ve/scielo.php?script=sci_arttext\&pid=S0798 72692009000100010

47. Ferreira IMMMG. Crescimento e qualidade da ostra (Crassostrea gigas) em viveiros da Ria Formosa sujeitos a diferentes condições de cultura e situações ambientais [Dis sertação]. Porto: Universidade do Porto, 2003. 73 p.

48. Angell CL. Crescimiento y mortalidad de la ostra de mangle cultivada (Crassostrea rhizophorae). Mem Fund La Salle Cien Nat. 1973;33(94-94):152-62.

49. Areias DLL. Efeito da salinidade e temperatura no assentamento da ostra Crassostrea gasar (Adanson, 1757) e indução da desova da ostra Crassostrea gigas (Thundberg, 179) Porto: Universidade de Porto, 2012. 74 p.

50. Guimarães IM, Antonio ÍG, Peixoto S, Olivera A. Influência da salinidade sobre a sobrevivência da ostra-do-mangue, Crassostrea rhizophorae. Arq Ci Mar. 2008;41(1):118-22. Disponível em: http://www.periodicos.ufc.br/arquivos decienciadomar/article/view/6084/4296

51. Brito Ld. Efeito da salinidade sobre o crescimento da ostra nativa Crassostrea sp. como subsídio ao desenvolvimento da maricultura de espécies nativas em mar aberto [Dis sertação]. Portal do Paraná PR: Universidade Federal do Paraná, 2008. 47 p.

52. Cáceres-Puig JI, Abasolo-Pacheco F, Mazón-Suástegui JM, Maeda-Martinez AN, Saucedo PE. Effect of temperature on growth and survival of Crassostrea corteziensis spat during late-nursery culturing at the hatchery. Aquac. 2007;272(1-4):417-22. doi:10.1016/j.aquaculture.2007.06.030

53. Villarroel E, Buitrago E, Lodeiros C. Identificación de factores ambientales que afectan al crecimiento y la supervivencia de Crassostrea rhizophorae (Mollusca: Bivalvia) bajo condiciones de cultivo suspendido en el Golfo de Cariaco, Venezuela. Rev Científica FCV-LUZ. 2004;14(1):28-35.

54. Brown JR, Hartwick EB. Influences of Temperature, Salinity and Available Food Upon Suspended Culture of the Pacific Oyster, Crassostrea gigas I. Absolute and Allometric Growth. Aquac. 1988;70(3):231-51. doi:10.1016/0044-8486(88)90099-3 
55. Lopes GR, Gomes, CHAM, Tureck, CR, Melo, CMR. Growth of Crassostrea gasar cultured in marine and estuary environments in Brazilian waters. Pesquisa Agropecuária Brasileira. 2013. 48(7): 975-82. http://dx.doi.org/10.1590/S0100-204X2013000800024

56. Nuñez MP, Lodeiros C, Ramirez E, Narváez N, Graziani C. Crecimiento y sobrevivencia de la ostra de mangle Crassostrea rhizophorae bajo condición de cultivo intermareal y submareal. Zootec Trop. 2010;28(2):239-54.

57. Chávez-Villalba J, Arreola-Lizárraga A, Burrola-Sánchez S, Hoyos-Chairez F. Growth, condition, and survival of the Pacific oyster Crassostrea gigas cultivated within and outside a subtropical lagoon. Aquac. 2010;300(1-4):128-36. doi:10.1016/j.aquaculture.2010.01.012

58. Góngora-Gómez AM, Hernández-Sepúlveda JA, García-Ulloa M, Domínguez-Orozco AL. Crecimiento del ostión Crassostrea gigas (Thunberg, 1795) cultivado en el estero La Piedra, Sinaloa, México. Avances Investig Agropec. 2012;16(2):91-104. Disponível em: http://www.redalyc.org/articulo.oa?id=83723532006

59. Márquez A, Lodeiros C, Semidey D, Carpio M, Graziani C. Crecimiento y supervivencia de la ostra perlífera Pinctada imbricata (Röding 1798), bajo diferentes sistemas de confi namiento en cultivo suspendido. Zootec Trop. 2011;29(3):337-51.

60. Ferretti PMA. Avaliação comparativa da sobrevivência e do crescimento de ostras da espécie Crassostrea gigas com o emprego de diferentes estruturas de cultivo [Monografia]. Florianópolis - SC: Universidade Federal de Santa Catarina, 2008. 68 p.

61. Valentim FT. Avaliação do crescimento de sementes de ostras Crassostrea gigas utilizando dois tipos de berçários, na Praia da Cerca, Guarapari - ES [Monografia]. Vitória, ES: Universidade Federal do Espírito Santo - UFES, 2005. 44 p.

62. Fonseca BPV. Efecto de la densidad de siembra y los factores ambientales en el cultivo de ostión japonés Crassostrea gigas en la península de Lucernilla, Navolato, Sinaloa [Dissertação]. Guase, Sinaloa, México: Instituto Politécnico Nacional, 2011. 103 p.

63. Maccacchero GB, Ferreira JF, Guzenski J. Influence of stocking density and culture management on growth and mortality of the mangrove native oyster Crassostrea sp. in southern Brazil. Biotemas. 2007;20(3):47-53. Disponível https://periodicos.ufsc.br/index.php/biotemas/article/viewFile/20668/18826

64. Antonio ÍG. Efeitos da salinidade e densidade de estocagem no crescimento e sobrevivência larval da ostra do mangue Crassostrea rhizophorae (Guilding, 1828) sob diferentes tempos de troca de água [Dissertação]. Recife-PE: Universidade Federal Rural de Pernambuco, 2007. 52 p.

65. Pereira OM, Machado IC, Henriques MB, Yamanaka N. Crescimento da ostra Crassostrea brasiliana semeada sobre tabuleiro em diferentes densidades na região estuarino-lagunar de Cananéia-SP $\left(25^{\circ} \mathrm{s}\right.$, 48 w). Bol Inst Pesca. 2001;27(2):163-74. https://www.pesca.sp.gov.br/27\%5B2\%5D-art_06.pdf

66. Levinton J, Doall M, Allam B. Growth and Mortality Patterns of the Eastern Oyster Crassostrea virginica in Impacted Waters in Coastal Waters in New York, USA. J Shellfish Res. 2013; 32(2):41727. doi:10.2983/035.032.0222

67. Díaz OD, Liñero-Arana I, Villafranca S, Allen T. Epizoic polychaetes (Annelida: Polychaeta) on Crassostrea rhizhophorae (Guilding, 1828) from la restinga lagoon, Margarita Island, Venezuela. Ecotrópicos. 2009. 22(1): 13-22.

68. Bonifácio PHdO. Polidorídeos (Polychaeta: Spionidae) em Crassostrea rhizophorae (Mollusca: Bivalvia) de cinco rios da costa pernambucana [Dissertação]. Recife - PE: Universidade Federal de Pernambuco, 2009. $47 \mathrm{p}$.

69. Pinto FMVS. Efeito de organis mos incrustantes sobre o crescimento e a sobrevivência de os tras nativas do gênero Crassostrea em um cultivo suspenso na Baía de Guaratuba (Paraná - Brasil) [Monografia]. Pontal do Paraná: Universidade Federal do Paraná, 2007. 53 p. Disponível em: http://hdl.handle.net/1884/36593

70. Littlewood DTJ, Marsbe LA. Predation on cultivated oysters, Crassostrea rhizophorae (Guilding ), by the polyclad turbellarian flatworm, Stylochus (Stylochus) frontalis Verrill. Aquac. 1990;88:145-50. 